\title{
Near-miss Injury of Multiple Carpometacarpal Joint Dislocations: A Report of Two Cases
}

Kow Ren Yi1 ${ }^{1}{ }^{\star}$, Zaharul Azri Mustapha Zakaria ${ }^{3}$, Ruben Jaya Kumar ${ }^{3}$, Low Chooi Leng ${ }^{4}$, Khairul Nizam Siron Baharom²

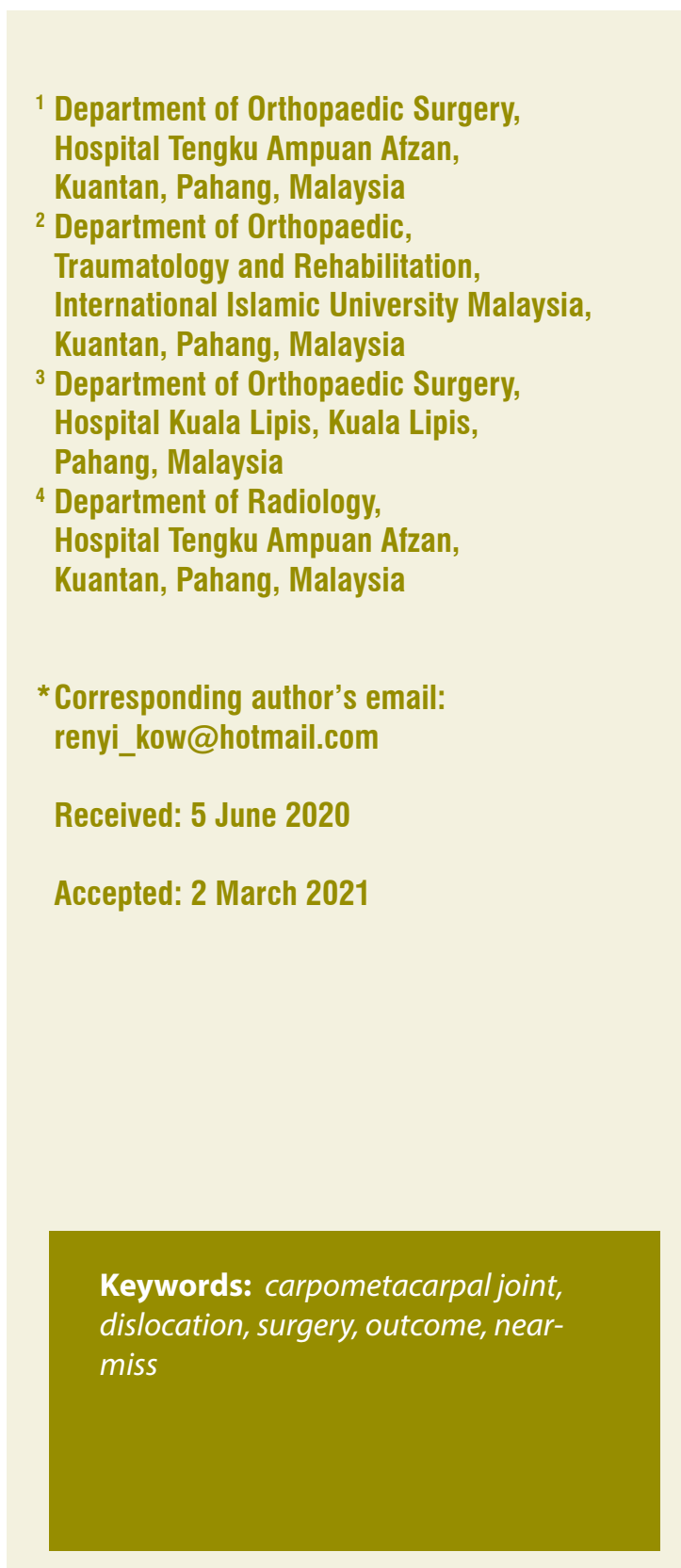

\section{ABSTRACT}

Multiple carpometacarpal joint (CMCJ) dislocations are rare and are easily missed. The anatomical configuration renders stability to the joints. As a result, a high-velocity impact is required to dislocate the joint. We present two cases of multiple carpometacarpal joint dislocations with different mechanisms of injury and their subsequent management at our centre. In case 1, a 29-year-old gentleman presented with acute dislocations of the CMCJs involving the right middle, ring, and little fingers. An open reduction and fixation with Kirschner wires were done to stabilize the dislocated CMCJs and he subsequently recovered. In case 2, a 25-year-old gentleman had the CMCJ dislocations detected late due to other more profound injuries. Partial arthrodesis was performed to address the instability of the CMCJs of the right index, middle and ring fingers. He subsequently recovered and returned to work 6-months postoperatively. The diagnosis of carpometacarpal joint dislocation can be easily missed especially when there is a concurrent distracting injury. It is crucial to maintain a high index of suspicion as early diagnosis and prompt treatment is paramount to yield a better outcome.

\section{INTRODUCTION}

Dislocations of multiple carpometacarpal (CMC) joints are uncommon (Bao et al., 2018). They account for less than one per cent of all hand traumas (Beekhuizen et al., 2018). The CMC joints are strengthened by multiple ligaments especially the dorsal and volar ligaments in addition to the anatomical saddle joint configuration, hence explaining 
the low incidence of the injury (Buren et al., 2016). Injury, when happens, usually affects the CMC joints of the ring and little fingers due to the looser ligamentous arrangement and more mobility as a result of the saddle joint configuration (Metikala et al., 2020). Anatomically, the pisiform bone and the hook of the hamate act as a volar buttress at the ulnar region, resulting in a higher incidence of dorsal CMC joint dislocations (Pundkare et al.,2015). Dorsal dislocations form $85 \%$ of all CMC joint dislocations with the 5th CMC joint being the most commonly dislocated one (Cobb et al., 2018; Nanno et al., 2007). Dislocations of the CMC joints can be classified based on the Pundkare Classification (Table 1) (Pundkare et al., 2018).

Table 1 Pundkare Classification of dislocations of the CMC joints (Pundkare et al.,2018)

\begin{tabular}{|l|l|l|l|}
\hline Type & Direction of dislocation & Involved metacarpals & Peculiarity \\
\hline A & Dorsal & 1 or more & All in the same direction \\
\hline B & Volar & 1 or more & All in the same direction \\
\hline C & Divergent: Dorsal and Volar & 2 or more & $\begin{array}{l}\text { At least two in the opposite } \\
\text { direction }\end{array}$ \\
\hline $\begin{array}{l}\text { To add } \\
1\end{array}$ & $\begin{array}{l}\text { Any of the above plus concomitant carpal } \\
\text { or metacarpal fracture }\end{array}$ & $\begin{array}{l}\text { Metacarpal dislocation with } \\
\text { associated fractures }\end{array}$ & Displaced fracture \\
\hline
\end{tabular}

CMC joint dislocations are often missed because of subtle radiographic changes and treating clinicians focusing on other more severe concomitant injuries. Henderson et al. (1987) reported that more than $70 \%$ of the CMC joint dislocations were missed at the accident and emergency department. In this case report, there are two cases of carpometacarpal joint dislocations with different mechanisms of injury and their subsequent management.

\section{CASE PRESENTATION}

\section{Case One}

$\mathrm{Mr} \mathrm{R}$, a 29-year-old male, presented with pain and swelling at his dominant right hand one week after punching another person in the face with his right closed fist in a fight at the prison. On examination, the swelling was localized at his right wrist with a limited range of movement of the wrist due to pain. Plain radiographs of his right wrist revealed dislocations of the $C M C$ joints involving the right middle, ring, and little fingers (Figure 1). 


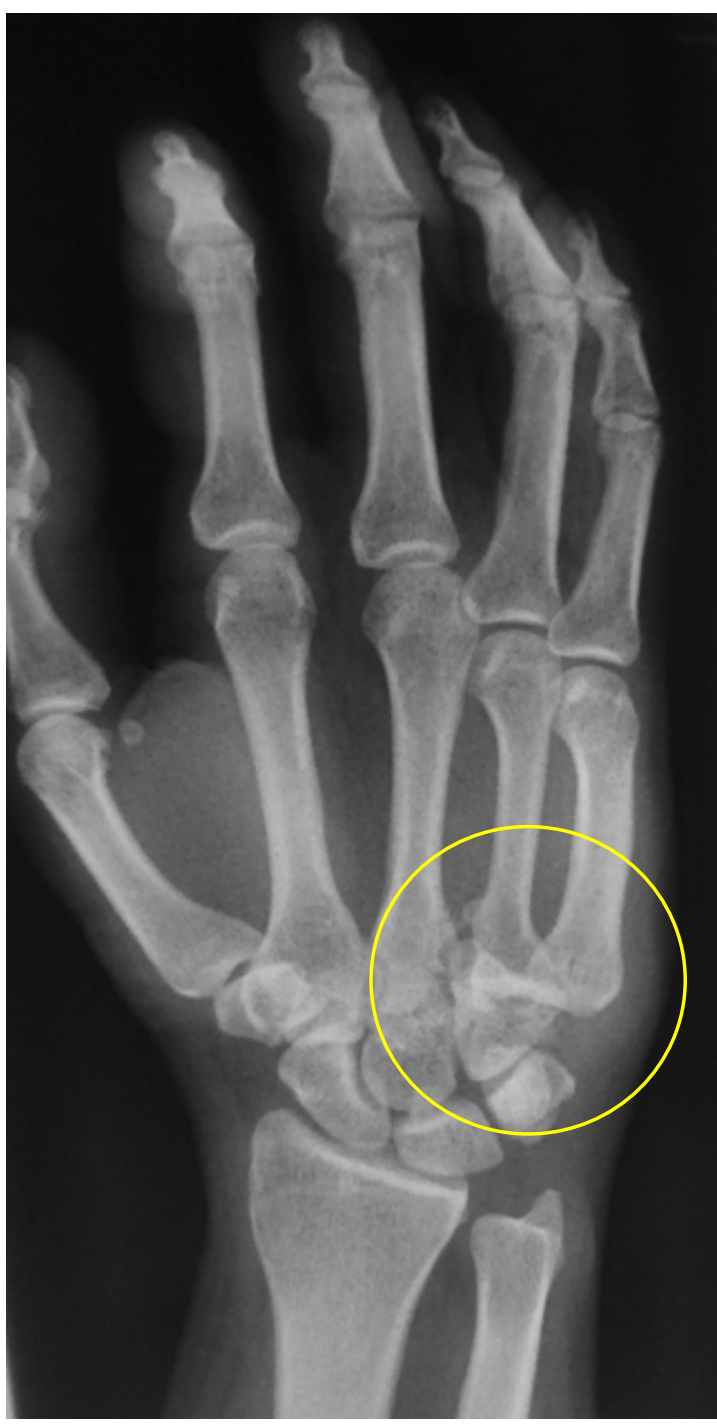

A

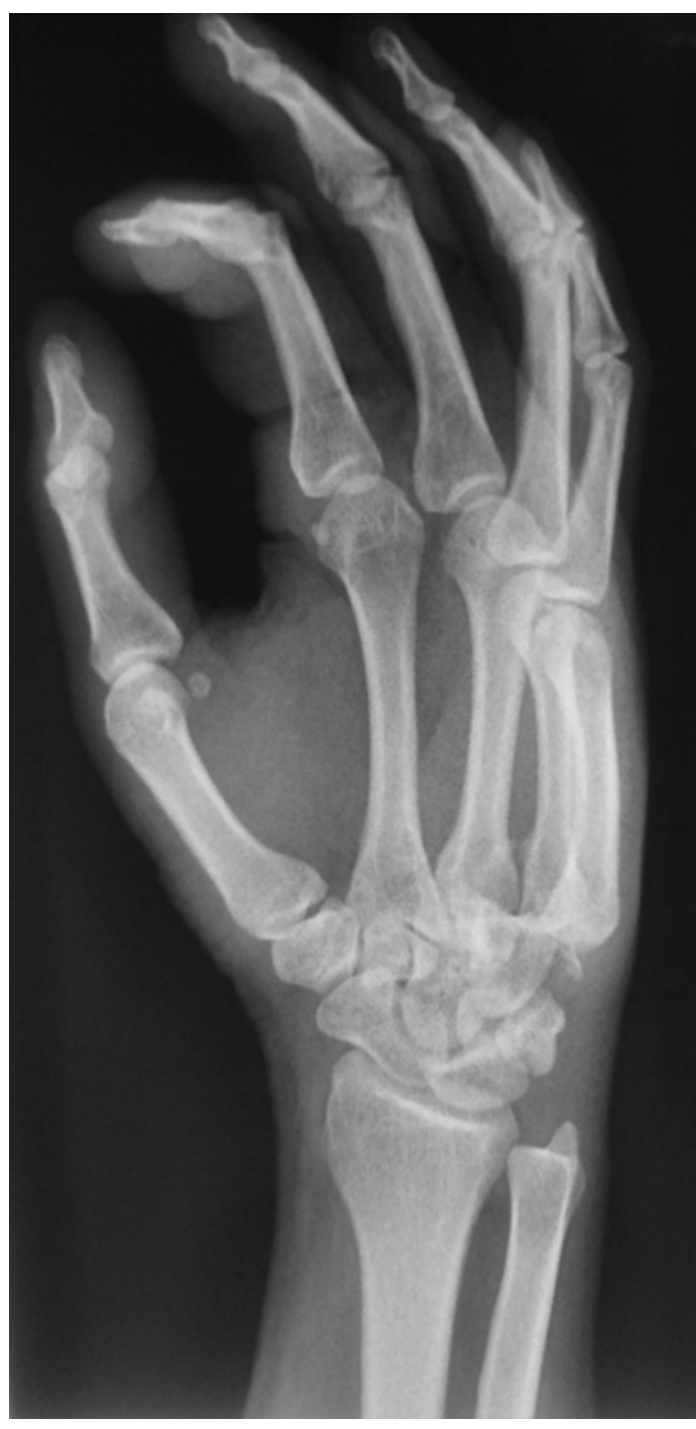

B

Figure 1 Antero-posterior (AP) (A) and oblique (B) plain radiographs of the right hand show the carpometacarpal (CMC) joint dislocations. Note that there is a disruption of the parallel ' $M$ lines' on the AP plain radiograph (yellow circle), hence raising the suspicion of CMC joint dislocations.

The initial trial of closed manipulative reduction was futile and an open reduction and fixation with Kirschner wires were then done to stabilize the dislocated CMC joints of the right middle, ring and little fingers (Figure 2 and Figure 3). Two longitudinal skin incisions were made, one overlying the second $C M C$ joint and the other one overlying the fourth $C M C$ joint to visualize and examine the stability of all four ulnar CMC joints (Figure 2). Post-operatively, the injured hand was protected with a volar slab for three weeks and then started on handgrip exercises. He subsequently recovered well without any complication. A handgrip test with a dynamometer showed that the right-hand grip strength was $75 \%$ compared to the contralateral normal left hand at six months post-operation. An attempt to reassess his handgrip strength was unsuccessful as he had defaulted the clinic follow-up after he was released from prison. 


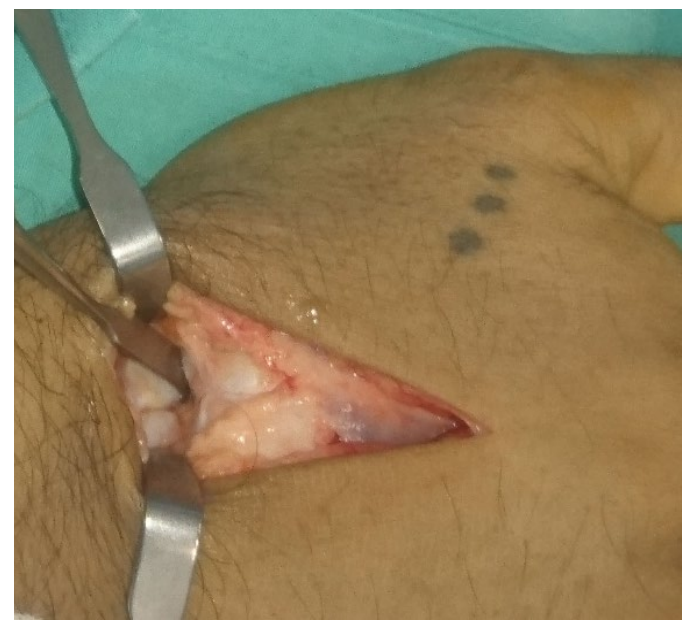

A

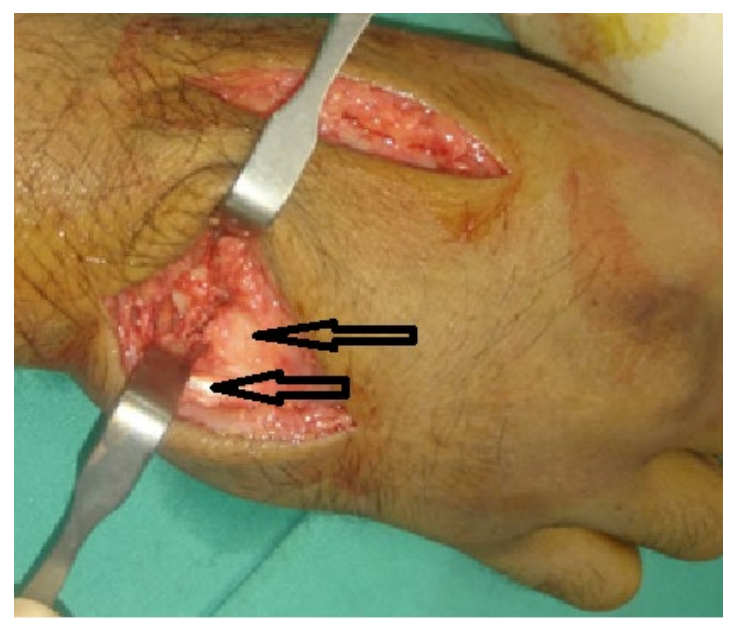

B

Figure 2 The $C M C$ joint of the index finger is examined with a McDonald elevator and appears to be stable (A). There is a gross displacement of the $C M C$ joints of the ring and little fingers (B - black arrows), with the metacarpal bone partially obscured by the extensor digitorum tendon. The CMC joint of the middle finger is unstable when stress is applied.

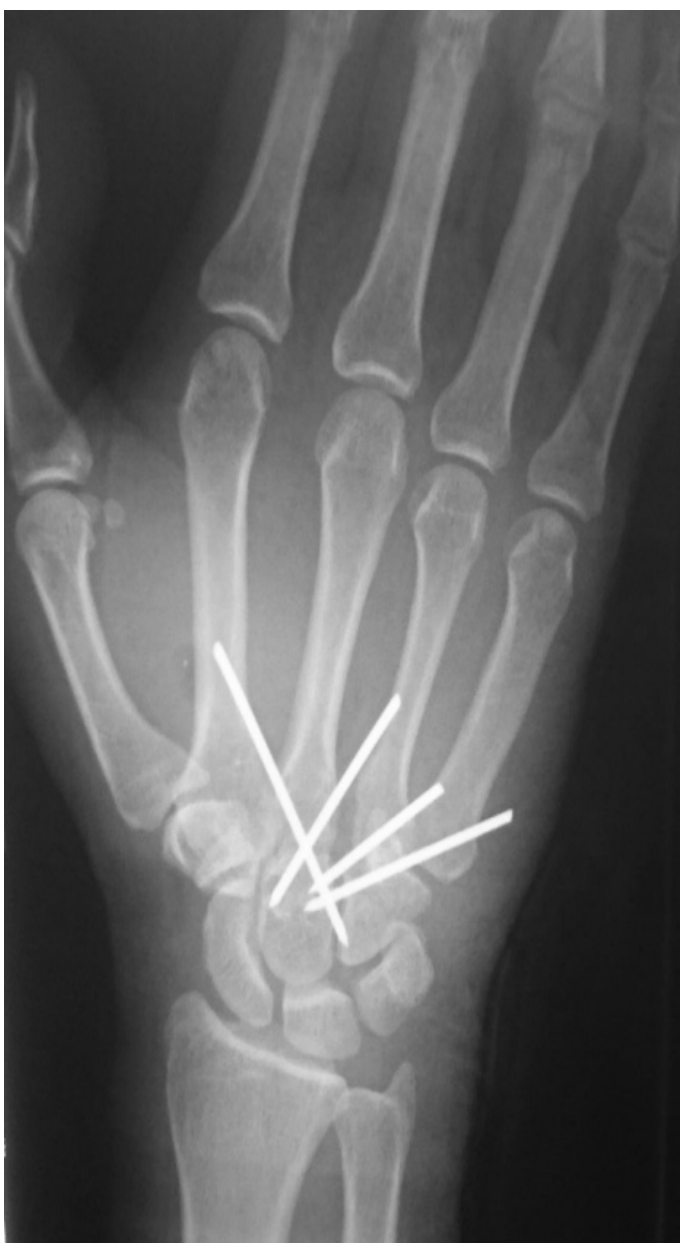

A

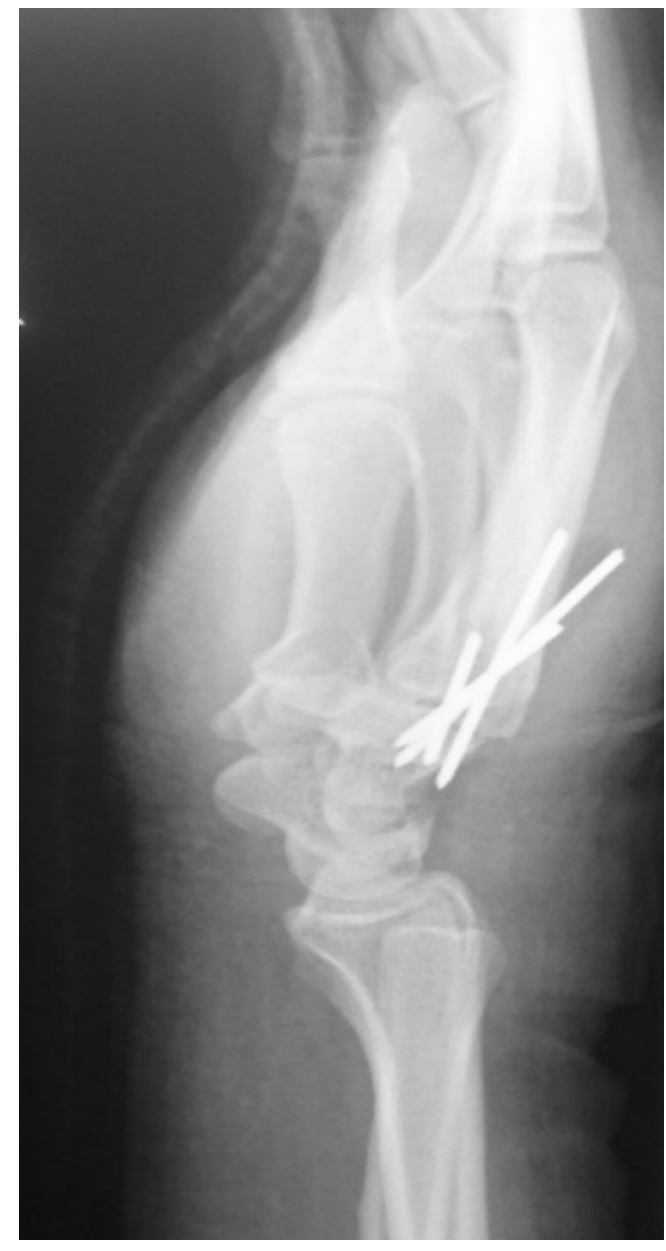

B

Figure 3 Plain radiographs of the right wrist ( $\mathrm{A}$ - anteroposterior view; B - lateral view) post-open reduction and Kirschner wiring show that the $C M C$ joints of the right middle, ring and little fingers are well-reduced. 


\section{Case Two}

$\mathrm{Mr} \mathrm{MH}$, a 25-year-old, right-hand dominant gentleman with no known medical illness, was involved in a motor vehicle accident and he sustained a cerebral concussion, an open comminuted fracture of the distal left radius Gustillo-Anderson grade $3 \mathrm{~A}$ and a closed mid-shaft fracture of his right radius and ulna. The diagnosis of CMC joint dislocations of the right index, middle and ring fingers had been missed during the initial clinical assessment. Wound debridement, cross-wrist external fixation for the distal left radius open fracture and an open reduction with small dynamic compression plating of the right radius and ulnar closed fractures were done. The CMC joint dislocations of the right hand were then revealed through plain radiographs of the right wrist on a post-operative day one, especially on the lateral view (Figure 4), which was not obvious on the initial anteroposterior and oblique view.

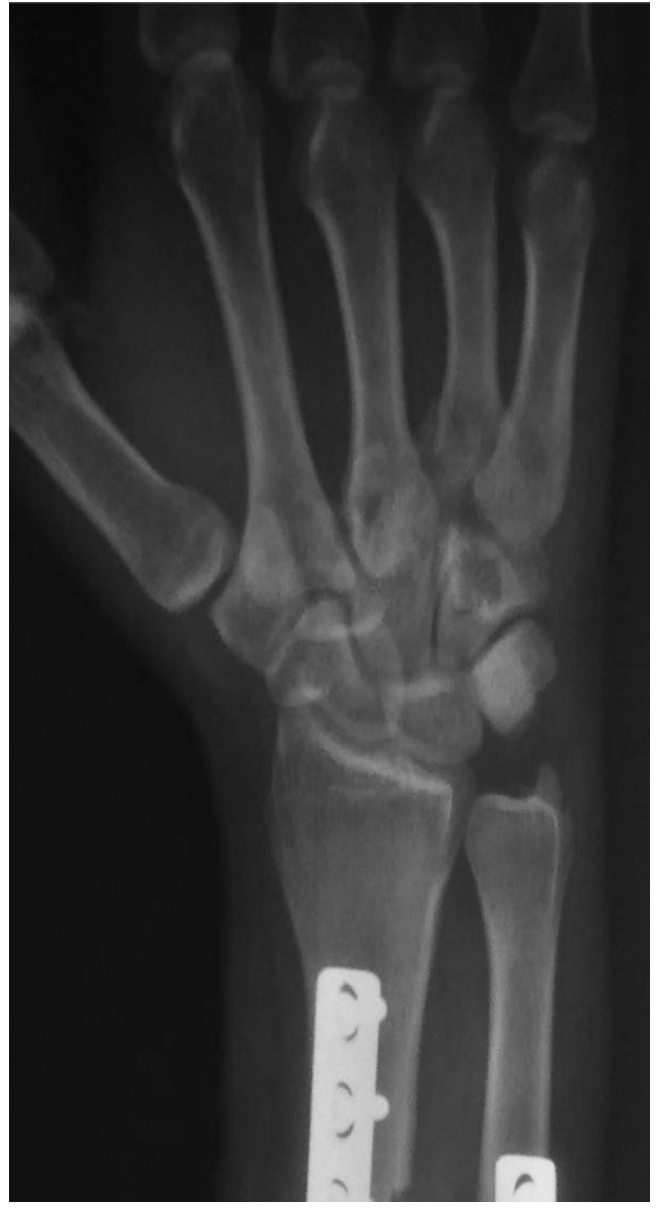

A

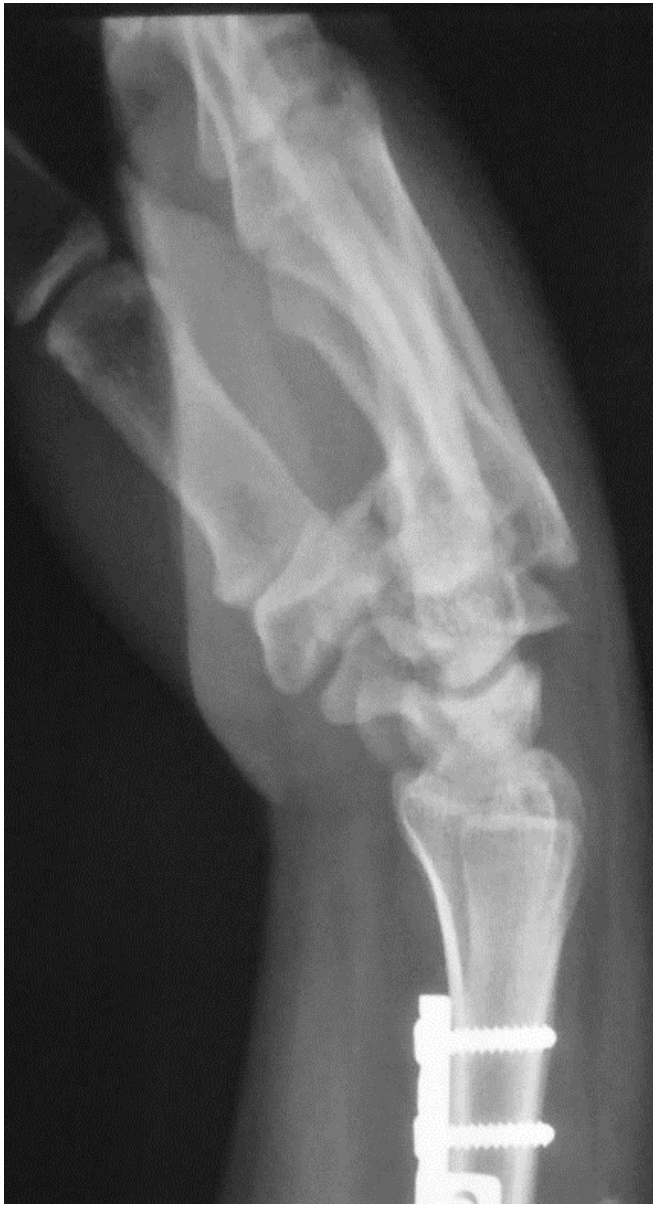

B

Figure 4 Plain radiographs of the right wrist ( - anteroposterior view; B - lateral view) show CMC joint dislocations of the index, middle and ring fingers

The patient opted and insisted for fixation of the right CMC joint dislocations during the surgery planned for the fixation of his left upper limb due to previous unpleasant general anaesthesia experience despite adequate counselling and explanation on associated risks were given. He underwent removal of the external fixator and variable-angle distal radius locking compression plating for his left distal end radius fracture 3 weeks after the first surgery due to an unfavourable soft tissue condition at his left forearm. Intra-operatively, the CMC joints of the right index, middle and ring fingers are grossly displaced with extensive fibrosis surrounding the joints, making the reduction difficult and challenging (Figure 5). 


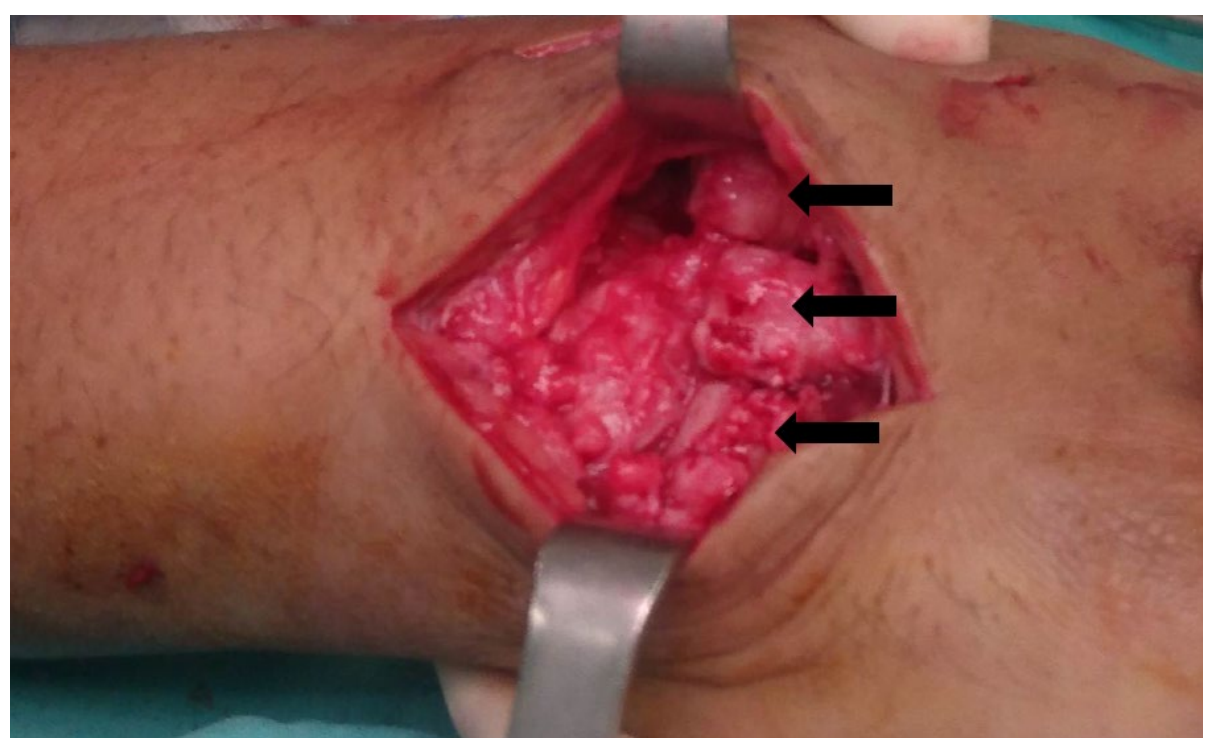

Figure 5 Intra-operatively, the CMC joints of the index, middle and ring fingers (arrows) are grossly displaced with extensive fibrosis surrounding the joint, making the reduction difficult.

The CMC joints were reduced, and they were fixed with two Kirschner wires. Partial arthrodesis was done by using a $4.0 \mathrm{~mm}$ screw and an iliac bone graft was used due to grossly unstable CMC joints (Figure 6). A volar slab was used for 3 weeks after the surgery for protection.

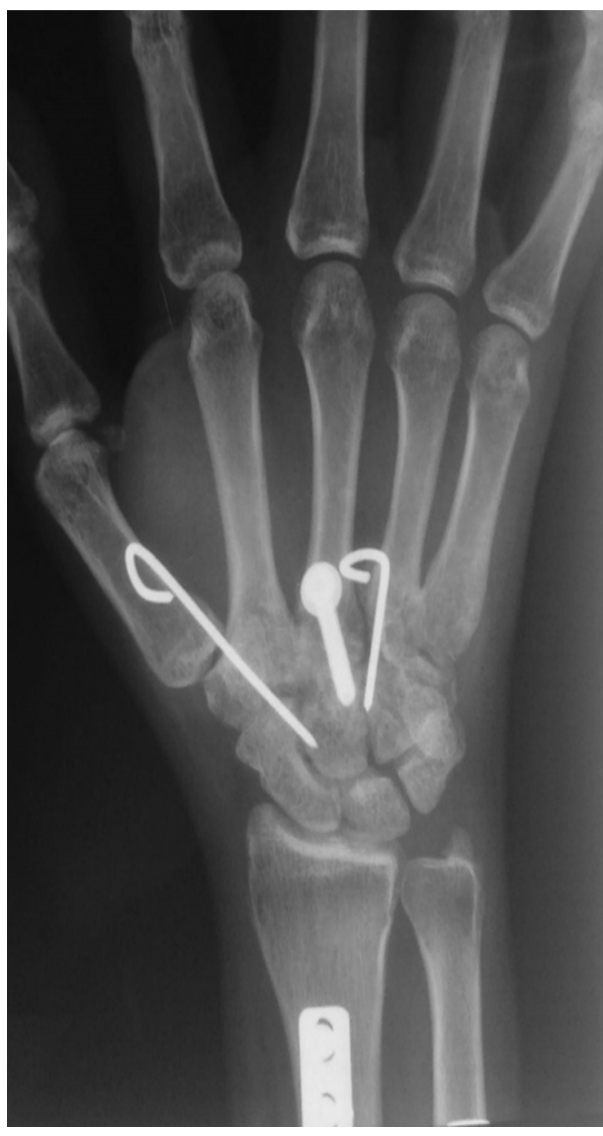

A

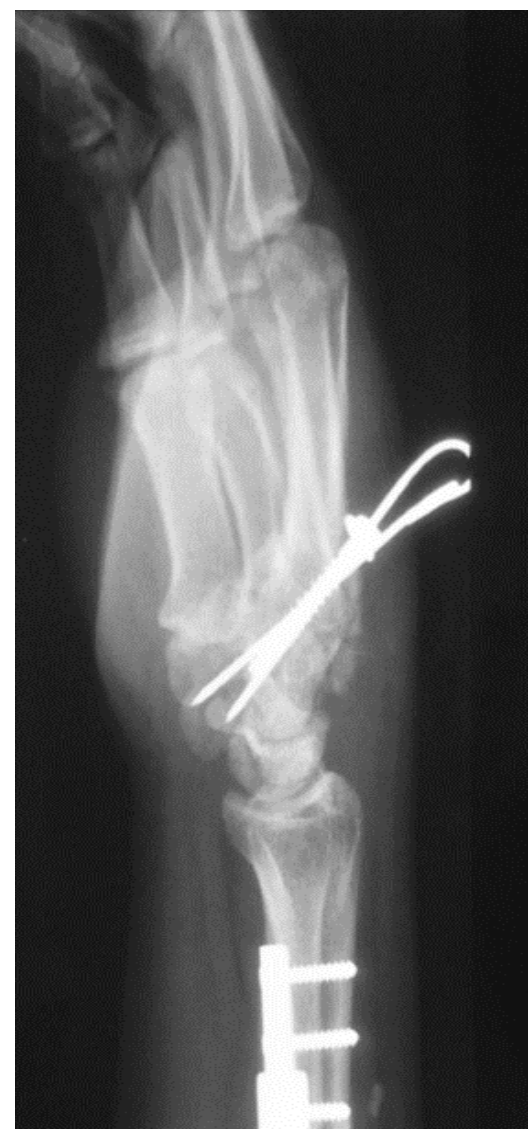

B

Figure 6 Plain radiographs ( $\mathrm{A}$ - anteroposterior view; B - lateral view) of the right wrist show that the dislocations are reduced, and partial arthrodesis is done with a $4.0 \mathrm{~mm}$ screw due to severely unstable CMC joints. 
The Kirschner wires are removed in the clinic setting at 3 weeks post-reduction (Figure 7). However, he refused the removal of the cancellous screw and he denied having symptoms of implant prominence.

He was able to return to work 6 months after the surgery. At 18 months after the surgery, his grip strength based on the handgrip dynamometer on the right hand was $40 \mathrm{~kg}$ with comparable grip strength on the left hand. He was able to flex his right wrist up to 45 degrees, extend up to 30 degrees with 10 degrees ulnar deviation and zero-degree radial deviation.

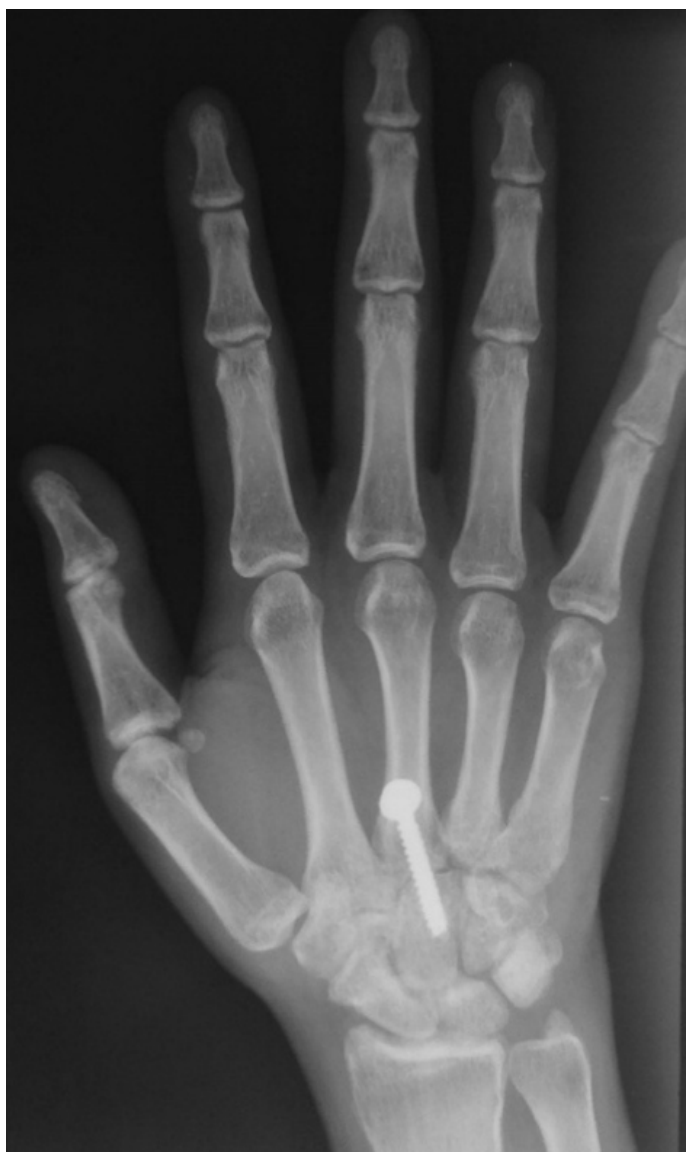

A

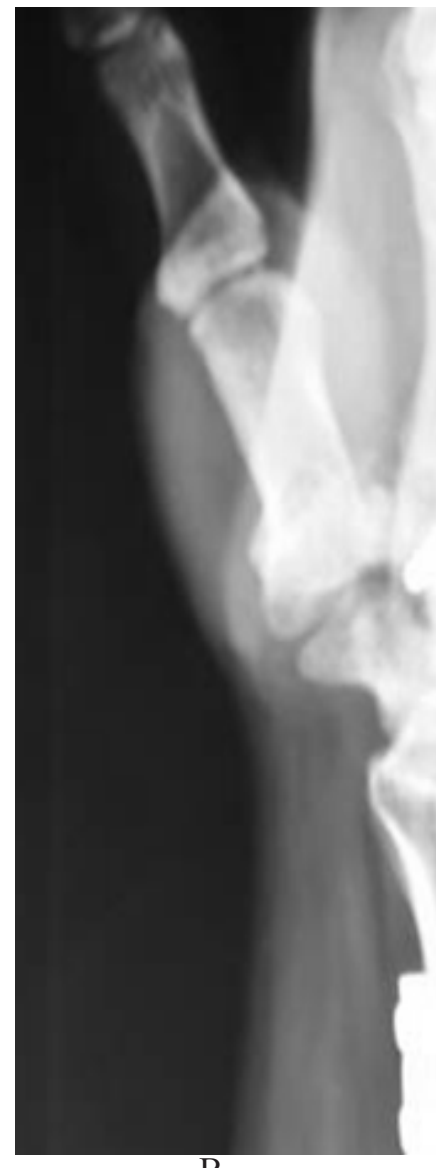

B

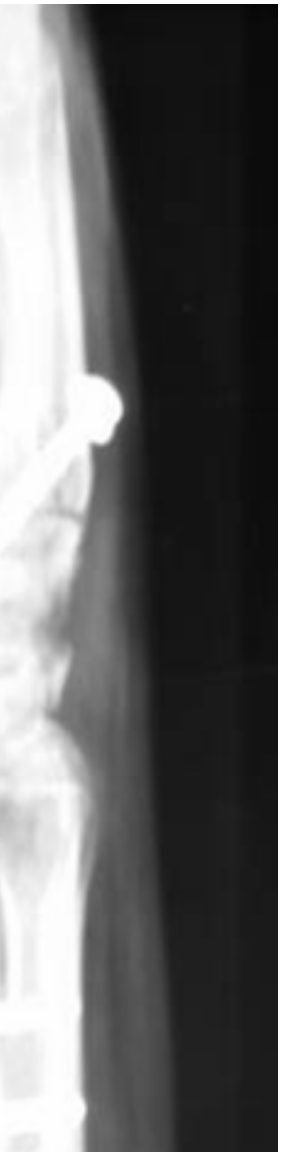

Figure 7 Plain radiographs of the right wrist (A - anteroposterior view; B - lateral view) show wellreduced CMC joints after the removal of Kirschner wires

\section{DISCUSSION}

Carpometacarpal joint dislocations are reported in the literature as isolated case reports or case series due to their rare incidence (Deshpande et al., 2017; Gil et al., 2017). The treatment varies based on the patient's presentation. It is generally agreed that early diagnosis and subsequent management, either conservative or surgical intervention, yield a better functional outcome (Tay et al., 2019; Zhang et al., 2015). In the acute setting (up to 2 weeks post-injury), closed manipulative reduction and splint immobilization of the CMC joints can be attempted. These patients should be monitored closely as there is a risk of secondary dislocation after the initial closed reduction and splinting. The risk of displacement is higher if the second and third CMC joints are involved, as a result of the dorsal pull of the extensor carpi radialis longus (ECRL) and extensor carpi radialis brevis (ECRB) tendons (Gil et al., 2017). Other methods such as closed reduction and percutaneous fixation 
with Kirschner wires as well as open reduction and fixation with Kirschner wires or plates have been reported to be successful (Tay et al., 2019; Telich-Tarriba et al., 2020).

In both of the patients, there is multiple CMC joints involvement, hence an open reduction is imperative to obtain a good reduction of the $C M C$ joints. In the first patient, fixation of the 3 ulnar CMC joints is made with Kirschner wires after an open reduction. The surgery for the second patient is complicated with extensive fibrosis surrounding the dislocated right second, third and fourth CMC joints, which is expected after a delay of 3 weeks. It is also unusual to have an intact fifth CMC joint when the other CMC joints are dislocated. After the reduction, fixation with Kirschner wires alone is unstable, hence a partial arthrodesis with a $4.0 \mathrm{~mm}$ screw and autologous iliac bone grafting is done. Based on previous case series, most of the CMC joint dislocations can be treated with K-wiring after reduction, either with closed or open reduction method and they generally produce a good long-term functional outcome. Arthrodesis of the CMC joint should be reserved for those patients with more severe injury or as a treatment for secondary osteoarthritis. Functional outcomes of patients who undergone arthrodesis after a CMC joint dislocation is not available in the literature as it is rarely done (Tay et al., 2019; Telich-Tarriba et al., 2020).

After an anatomic restoration of the CMC joints, good outcomes can be expected. The range of movement of the CMC joints will be compensated by other joints. The main goal of treatment is to obtain a pain-free wrist and to regain grip strength which can be achieved by undergoing extensive rehabilitation and physiotherapy post-operatively. We advocate for our patients to start handgrip exercises by using plasticine at 3 weeks post-reduction. Active range of movement exercises was started at 6 weeks post-reduction and subsequently passive range of movement exercises at 2 months post-surgery. Despite the delayed treatment, both of our patients have regained good functional handgrip strength by following this rehabilitation protocol. Generally, patients are advised for gradual mobilization of the fingers and CMC joints after four to six weeks of immobilization, followed by removal of the implants and more intense physiotherapy at eight to ten weeks post-operatively (Tay et al., 2019; Telich-Tarriba et al., 2020).

\section{CONCLUSION}

Carpometacarpal joint dislocation is an uncommon injury of the hand that is easily missed. Early diagnosis is the key to achieve a good functional outcome. Understanding the mechanism of injury with a high index of suspicion is the sine qua non in making an early diagnosis of carpometacarpal joint dislocation.

\section{CONFLICT OF INTEREST}

The authors declare that they have no competing interests in publishing this case.

\section{CONSENTS}

Written consent was obtained from the patients to publish the case. A copy of the written consent is available for review by the Chief Editor.

\section{ACKNOWLEDGEMENTS}

This publication is supported by SASMEC @ IIUM Research Grant (SRG). 


\section{REFERENCES}

Bao, B., Zhu, H., \& Zheng, X. (2018). Plate versus Kirschner wire fixation in treatment of fourth and fifth carpometacarpal fracture-dislocations: A retrospective cohort study. International Journal of Surgery, 52, 293 - 296. https://doi.org/10.1016/j.ijsu.2018.01.052

Beekhuizen, S., de Witte, P. B., Rutgers, M., \& Ohanis, D. (2018). Isolated ulnopalmar dislocation of the fifth carpometacarpal joint. BMJ Case Reports, bcr2018225363. https:doi. org/10.1136/bcr-2018-225363

Buren, C., Gehrmann, S., Kaufmann, R., Windolf, J., \& Logters, T. (2016). Management algorithm for index through small finger carpometacarpal fracture dislocations. European Journal of Trauma and Emergency Surgery, 42 (1), 37 - 42. https://doi.org/10.1007/S00068015-0611-z

Cobb, W. A., Dingle, L., Adami, R. Z., \& Rodrigues, J. (2018). Management of fracture-dislocations of the little finger carpometacarpal joint: $A$ systematic review. Journal of Hand Surgery (European Volume), 43 (5), 530 - 538. https:// doi.org/10.1177/1753193417752317

Deshpande, M., Doomra, T., \& Nadkarni, S. (2017). Multiple carpometacarpal dislocations of the hand - an uncommon variant: $A$ rare case. Journal of Hand Surgery (Asian Pacific Volume), 22 (3), 371 - 375. https://doi. org/10.1142/S0218810417720261

Gil, J. A., Goodman, A. D., \& Starr, A. (2017). Coronal hamate fracture associated with carpometacarpal dislocations of all of the fingers: Review of the literature and case report. Hand, 12 (5), NP51 - NP54. https:// doi.org/10.1177/1558944716668860

Henderson, J. J., \& Arafa, M. A. (1987). Carpometacarpal dislocation. An easily missed diagnosis. The Journal of Bone and Joint Surgery, 69 (2), 212 - 214. https://doi. org/10.1302/0301-620X.69B2.3818751
Metikala, S., \& Herickhoff, P. (2020). isolated dorsal dislocations of the fourth and fifth carpometacarpal joints: A case report and review of literature. Cureus, 12 (12), e12310. https:// doi.org/10.7759/cureus.12310

Nanno, M., Sawaizumi, T., \& Ito, H. (2007). Dorsal fracture dislocations of the second and third carpometacarpal joints. Journal of hand Surgery (European Volume), 32 (5), 597 - 598. https://doi.org/10.1016/j.jhse.2007.03.008

Pundkare,G.T., \&Patil, A. M. (2015). Carpometacarpal joint fracture dislocation of second to fifth finger. Clinics in Orthopedic Surgery, 7 (4), 430 - 435. https://doi.org/10.4055/ cios.2015.7.4.430

Pundkare, G. T., \& Deshpande, S. S. (2018). Proposal for a radiological classification system for carpo-metacarpal joint dislocations with or without fractures. Malaysian Orthopaedic Journal, 12 (2), 42 - 46. https://doi. org/10.5704/MOJ.1807.008

Tay, S. C., Leow, M. Q. H., \& Tan, E. S. (2019). Use of dorsal buttress plate fixation for ulnar carpometacarpal joint fracture dislocations for early mobilization: Outcomes of 11 cases. Musculoskeletal Surgery, 103 (1), 77 - 82. https://doi.org/10.1007/s12306-018-0571-7

Telich-Tarriba, J. E., Guevara-Valmana, O. I., Navarro-Barquin, D. F., \& Victor-Baldin, A. (2020). Carpometacarpal joint dislocations: Management and long-term outcomes at a specialised hand surgery center in Latin America. Plastic Surgery, 28 (4), 210 - 214. https://doi.org/10.1177/2292550320933690

Zhang, C., Wang, H., Liang, C., Yu, W., Li, Y., Shang, R., Huang, C., \& Huang, C. (2015). the effect of timing on the treatment and outcome of combined fourth and fifth carpometacarpal fracture dislocations. Journal of Hand Surgery, 40 (11), 2169 - 2175. https://doi. org/10.1016/j.jhsa.2015.07.017 
